\title{
ON DISCRETE ANALOGUES OF NONLINEAR IMPLICIT DIFFERENTIAL EQUATIONS
}

\author{
PHAM KY ANH AND LE CONG LOI
}

Received 16 February 2005; Revised 26 September 2005; Accepted 27 September 2005

This paper deals with some classes of nonlinear implicit difference equations obtained via discretization of nonlinear differential-algebraic or partial differential-algebraic equations. The unique solvability of discretized problems is proved and the compatibility between index notions for nonlinear differential-algebraic equations and nonlinear implicit difference equations is studied.

Copyright (c) 2006 P. K. Anh and L. C. Loi. This is an open access article distributed under the Creative Commons Attribution License, which permits unrestricted use, distribution, and reproduction in any medium, provided the original work is properly cited.

\section{Introduction}

The importance of implicit difference equations (IDEs) seems to flow from two sources. First, in real world situations it has been found that many problems are modeled by singular discrete systems, such as the Leslie population growth model, the Leontief dynamic model of multisector economy, singular discrete optimal control problems and so forth. Second, implicit discrete systems appear in a natural way of using discretization techniques for solving differential-algebraic equations (DAEs) and partial differential-algebraic equations (PDAEs).

Recently $[1,2,6]$, a class of implicit difference equations, called index-1 IDEs has been investigated. The solvability of initial-value problems (IVPs) as well as boundary-value problems (BVPs) associated with index-1 IDEs has been studied. In [1] a connection between linear index-1 DAEs and linear index-1 IDEs has been revealed. In particular, the compatibility between index notions for linear index-1 DAEs and linear index-1 IDEs has been established.

Until now, we have not found any results on the unique solvability of nonlinear implicit difference systems obtained via discretization by using explicit schemes for nonlinear DAEs and PDAEs. This problem will be studied in the paper.

The paper is organized as follows. In Section 2 we show that the explicit Euler method applied to nonlinear index-1 DAEs leads to nonlinear index-1 IDEs. Moreover, the 
convergence of the explicit Euler method for nonlinear index-1 DAEs is established. The results of this section are a "nonlinear version" of the corresponding results in [1]. Section 3 deals with the unique solvability of a discretized problem for degenerated parabolic equations. In Section 4 two numerical examples are given and finally Section 5 summarizes the main results of this work.

\section{Compatibility of index notions for nonlinear DAEs and IDEs}

According to Griepentrog and März [5], a nonlinear DAE

$$
f\left(x^{\prime}(t), x(t), t\right)=0, \quad t \in J:=\left[t_{0}, T\right],
$$

where the function $f: \mathbb{R}^{m} \times \mathbb{R}^{m} \times J \rightarrow \mathbb{R}^{m}$ is continuous in $t$ and continuously differentiable in the first two variables, is said to be of index- 1 if

(i) the null-space $\operatorname{Ker}(\partial f / \partial y)(y, x, t) \equiv \mathcal{N}(t)$ does not depend on $y, x \in \mathbb{R}^{m}$, and there exists a smooth projection $Q \in C^{1}\left(J, \mathbb{R}^{m \times m}\right)$ such that

$$
Q^{2}(t)=Q(t) ; \quad \operatorname{Im} Q(t)=\mathcal{N}(t) \quad \forall t \in J .
$$

(ii) the matrix $G(y, x, t):=(\partial f / \partial y)(y, x, t)+(\partial f / \partial x)(y, x, t) Q(t)$ is nonsingular $\forall y$, $x \in \mathbb{R}^{m}$ and $\forall t \in J$.

Together with (2.1) we consider a nonlinear IDE

$$
f_{n}\left(x_{n+1}, x_{n}\right)=0 \quad(n \geq 0),
$$

where the functions $f_{n}: \mathbb{R}^{m} \times \mathbb{R}^{m} \rightarrow \mathbb{R}^{m}$ are supposed to be continuously differentiable. We recall the following definition.

Definition 2.1 ([2, Definition 3.2]). Equation (2.3) is called an index-1 IDE if

(i) the subspaces $\mathcal{N}_{n}:=\operatorname{Ker}\left(\partial f_{n} / \partial y\right)(y, x)$ are independent of $y, x \in \mathbb{R}^{m}$ and have the same dimension, that is, $\operatorname{dim} \mathcal{N}_{n}=m-r$ for some integer $r$ between 1 and $m-1$,

(ii) the matrices $G_{n}(y, x):=\left(\partial f_{n} / \partial y\right)(y, x)+\left(\partial f_{n} / \partial x\right)(y, x) Q_{n-1, n}$ are nonsingular for all $y, x \in \mathbb{R}^{m}$ and $n \geq 0$, where the so-called connecting operators $Q_{n-1, n}$ are defined as follows.

Let $Q_{n-1}$ and $Q_{n}$ be arbitrary projections onto subspaces $\mathcal{N}_{n-1}$ and $\mathcal{N}_{n}$, respectively. Then $Q_{n-1}=V_{n-1} \widetilde{Q} V_{n-1}^{-1}$ and $Q_{n}=V_{n} \widetilde{Q} V_{n}^{-1}$, where $V_{n-1}, V_{n}$ are nonsingular matrices and $\widetilde{Q}=\operatorname{diag}\left(O_{r}, I_{m-r}\right)$. Here $O_{r}$ and $I_{m-r}$ stand for zero and identity matrices, respectively. We define an operator connecting two subspaces $\mathcal{N}_{n-1}$ and $\mathcal{N}_{n}$ as $Q_{n-1, n}=$ $V_{n-1} \widetilde{Q} V_{n}^{-1}$. For definiteness, we put $\mathcal{N}_{-1}:=\mathcal{N}_{0} ; Q_{-1}:=Q_{0}$ and $V_{-1}:=V_{0}$.

It can be verified (cf. [2]) that the matrices $G_{n}(y, x)$ are nonsingular if and only if

$$
S_{n}(y, x) \cap \mathcal{N}_{n-1}=\{0\} \quad \forall y, x \in \mathbb{R}^{m} ; \forall n \geq 0,
$$

where, as in the DAE case, $S_{n}(y, x)$ denotes the set

$$
\left\{\xi \in \mathbb{R}^{m}: \frac{\partial f_{n}}{\partial x}(y, x) \xi \in \operatorname{Im} \frac{\partial f_{n}}{\partial y}(y, x)\right\} .
$$


Since condition (2.4) does not depend on the choice of connecting operators, the correctness of the index-1 notion for nonlinear IDEs is guaranteed.

Now we discretize (2.1) by the explicit Euler scheme, namely

$$
f\left(\frac{x_{n+1}-x_{n}}{\tau}, x_{n}, t_{n}\right)=0, \quad n=\overline{0, N-1}
$$

where $t_{n}=t_{0}+n \tau ; \tau:=\left(T-t_{0}\right) / N, n=\overline{0, N}$. The following theorem ensures the compatibility of index notions for DAE (2.1) and IDE (2.6).

Theorem 2.2. Suppose the DAE (2.1) is of index-1 and the matrices $G^{-1}(y, x, t)$ and $(\partial f /$ $\partial x)(y, x, t)$ are uniformly bounded. Then for sufficiently small $\tau$, the discretized equation (2.6) is also an index-1 IDE.

Proof. For the proof of the theorem we first reduce (2.6) to its normal form (2.3). Then we will show that $(2.3)$ is of index-1 by verifying all the conditions of Definition 2.1 . Let the $\operatorname{DAE}(2.1)$ be of index-1. Then the null-space $\mathcal{N}(t)=\operatorname{Ker}(\partial f / \partial y)(y, x, t)$ does not depend on $y, x \in \mathbb{R}^{m}$ and is smooth in $t$. In particular, $\operatorname{dim} \mathcal{N}(t) \equiv m-r$ for some integer $r$ between 1 and $m-1$. Further, the matrix $G(y, x, t):=(\partial f / \partial y)(y, x, t)+(\partial f / \partial x)(y, x, t) Q(t)$, where $Q(t)=V(t) \tilde{Q} V^{-1}(t)$ is a smooth projection on $\mathcal{N}(t)$, is nonsingular.

To reduce (2.6) to $(2.3)$, we put $f_{n}(y, x):=f\left((y-x) / \tau, x, t_{n}\right)(n=\overline{0, N-1})$. First observe that

$$
\begin{gathered}
\frac{\partial f_{n}}{\partial y}(y, x)=\frac{1}{\tau} \frac{\partial f}{\partial y}\left((y-x) / \tau, x, t_{n}\right) \\
\frac{\partial f_{n}}{\partial x}(y, x)=\frac{\partial f}{\partial x}\left((y-x) / \tau, x, t_{n}\right)-\frac{1}{\tau} \frac{\partial f}{\partial y}\left((y-x) / \tau, x, t_{n}\right) .
\end{gathered}
$$

Clearly, $\operatorname{Ker}\left(\partial f_{n} / \partial y\right)(y, x)=\operatorname{Ker}(\partial f / \partial y)\left((y-x) / \tau, x, t_{n}\right)=\mathcal{N}\left(t_{n}\right) \equiv \mathcal{N}_{n}$. Let $P(t):=I-$ $Q(t) ; Q_{n}:=Q\left(t_{n}\right) ; P_{n}:=P\left(t_{n}\right) ; V_{n}:=V\left(t_{n}\right), n \geq 0$ and $\mathcal{N}_{-1}:=\mathcal{N}_{0} ; Q_{-1}:=Q_{0} ; V_{-1}:=V_{0}$. We define connecting operators $Q_{n-1, n}=V_{n-1} \widetilde{Q} V_{n}^{-1}, n \geq 0$. To prove the index-1 property of (2.6) we have to verify the nonsingularity of the matrix

$$
\bar{H}_{n}(y, x):=\frac{\partial f_{n}}{\partial y}(y, x)+\frac{\partial f_{n}}{\partial x}(y, x) Q_{n-1, n} \equiv \frac{1}{\tau} H_{n}(y, x)
$$

where

$$
H_{n}(y, x):=\frac{\partial f}{\partial y}\left((y-x) / \tau, x, t_{n}\right)+\left[\tau \frac{\partial f}{\partial x}\left((y-x) / \tau, x, t_{n}\right)-\frac{\partial f}{\partial y}\left((y-x) / \tau, x, t_{n}\right)\right] Q_{n-1, n} .
$$

Letting $\bar{G}(y, x, t):=(\partial f / \partial y)(y, x, t)+\tau(\partial f / \partial x)(y, x, t) Q(t)$ and using the relation

$$
Q(t)=G^{-1}(y, x, t) \frac{\partial f}{\partial x}(y, x, t) Q(t),
$$


4 On discrete analogues of nonlinear implicit differential equations

we find

$$
\begin{aligned}
\bar{G}(y, x, t) & =G(y, x, t)-(1-\tau) \frac{\partial f}{\partial x}(y, x, t) Q(t) \\
& =G(y, x, t)\left[I-(1-\tau) G^{-1}(y, x, t) \frac{\partial f}{\partial x}(y, x, t) Q(t)\right] \\
& =G(y, x, t)[I-(1-\tau) Q(t)] \\
& =G(y, x, t)(P(t)+\tau Q(t)) .
\end{aligned}
$$

From the identity $(P(t)+\tau Q(t))^{-1} \equiv(1 / \tau)(\tau P(t)+Q(t))$, it follows

$$
\bar{G}^{-1}(y, x, t)=\frac{1}{\tau}(\tau P(t)+Q(t)) G^{-1}(y, x, t) .
$$

Now we will express $H_{n}(y, x)$ in terms of $G\left((y-x) / \tau, x, t_{n}\right)$ and projections $P_{n}, Q_{n}$. Observing that $(\partial f / \partial y)\left((y-x) / \tau, x, t_{n}\right) Q_{n}=0$ and $Q_{n-1, n}-Q_{n}=\left(V_{n-1}-V_{n}\right) \tilde{Q} V_{n}^{-1}$, after a short computation we find

$$
\begin{aligned}
H_{n}(y, x)=\bar{G}( & \left.(y-x) / \tau, x, t_{n}\right) \\
\times & \left\{I+\left[\left(\tau P_{n}+Q_{n}\right) G^{-1}\left((y-x) / \tau, x, t_{n}\right) \frac{\partial f}{\partial x}\left((y-x) / \tau, x, t_{n}\right)-P_{n}\right]\right. \\
& \left.\times\left(V_{n-1}-V_{n}\right) \tilde{Q} V_{n}^{-1}\right\} .
\end{aligned}
$$

By assumption, the matrices $G^{-1}\left((y-x) / \tau, x, t_{n}\right)$ and $(\partial f / \partial x)\left((y-x) / \tau, x, t_{n}\right)$ are uniformly bounded. Further, $P(t), Q(t)$ and $V^{-1}(t)$ are continuous, hence they are uniformly bounded on $J$. The smoothness of $V(t)$ on the compact segment $J$ implies that $\left\|V_{n-1}-V_{n}\right\| \leqslant c_{1} \tau$, where $c_{1}=\max _{t \in J}\left\|V^{\prime}(t)\right\|$. Thus the norm of the matrix

$$
M_{n}(y, x):=\left[\left(\tau P_{n}+Q_{n}\right) G^{-1}\left((y-x) / \tau, x, t_{n}\right) \frac{\partial f}{\partial x}\left((y-x) / \tau, x, t_{n}\right)-P_{n}\right]\left(V_{n-1}-V_{n}\right) \widetilde{Q} V_{n}^{-1}
$$

will be bounded by $c \tau$, where the constant $c$ is determined by the bounds of $G^{-1}, \partial f / \partial x$, $P, Q, V^{-1}$ and $V^{\prime}$. This fact implies the nonsingularity of the matrix

$$
\bar{H}_{n}(y, x)=\frac{1}{\tau} \bar{G}\left((y-x) / \tau, x, t_{n}\right)\left(I+M_{n}(y, x)\right),
$$

provided $\tau<\tau_{0}:=1 / c$. The proof of Theorem 2.2 is complete.

Now for finding a solution of (2.1), satisfying the initial condition

$$
P\left(t_{0}\right)\left(x\left(t_{0}\right)-x^{0}\right)=0
$$

we use the explicit Euler method, that is, we seek for a solution of (2.6) satisfying condition

$$
P_{0}\left(x_{0}-x^{0}\right)=0
$$


THEOREM 2.3. Under the assumptions of Theorem 2.2, the explicit Euler method applied to the IVP (2.1), (2.16) does converge.

Proof. The proof is divided into three steps. First, the H'adamard theorem is used for decomposing (2.1) into a system of an inherent ODE and an algebraic constraint. The second step is devoted to the similar decomposition for the discretized equation (2.6). The last step deals with the convergence of the explicit Euler method.

Step 1. Since $Q(t)$ is a projection onto $\mathcal{N}(t)=\operatorname{Ker}(\partial f / \partial y)(y, x, t)$ it implies

$$
f(y, x, t)-f(P(t) y, x, t)=\int_{0}^{1} \frac{\partial f}{\partial y}(s y+(1-s) P(t) y, x, t) Q(t) y d s=0, \quad \forall y, x \in \mathbb{R}^{m} ; \forall t \in J .
$$

Thus the IVP (2.1), (2.16) is reduced to the problem

$$
\begin{gathered}
f\left(P(t) x^{\prime}(t), P(t) x(t)+Q(t) x(t), t\right)=0, \quad t \in J, \\
P\left(t_{0}\right) x\left(t_{0}\right)=P\left(t_{0}\right) x^{0} .
\end{gathered}
$$

Putting $u(t):=P(t) x(t)$ we come to the equivalent IVP

$$
\begin{gathered}
f\left(P(t) x^{\prime}(t), u(t)+Q(t) x(t), t\right)=0, \quad t \in J, \\
u\left(t_{0}\right)=P\left(t_{0}\right) x^{0} .
\end{gathered}
$$

To establish the convergence of the explicit Euler method, we will treat the DAE (2.20) in a slightly different way than that of $[5,9]$. Since $P(t)$ and $Q(t)$ are smooth projections and $\operatorname{dim}(\operatorname{Im} P(t))=r, \operatorname{dim}(\operatorname{Im} Q(t))=m-r, \forall t \in J$, there exist linear homeomorphisms

$$
\xi_{t}: \mathbb{R}^{r} \longrightarrow \operatorname{Im} P(t), \quad \zeta_{t}: \mathbb{R}^{m-r} \longrightarrow \operatorname{Im} Q(t)
$$

such that $\xi_{t}$ and $\zeta_{t}$ depend continuously on $t \in J$.

For fixed $\bar{u} \in \mathbb{R}^{m}$ and $t, t_{1}, t_{2} \in J$ we consider an operator $F_{t ; \bar{u} ; t_{1}, t_{2}}: \mathbb{R}^{m} \rightarrow \mathbb{R}^{m}$ mapping every $z=\left(z_{1}^{T}, z_{2}^{T}\right)^{T} \in \mathbb{R}^{m}$, where $z_{1} \in \mathbb{R}^{r}$ and $z_{2} \in \mathbb{R}^{m-r}$, into $f\left(\xi_{t_{1}} z_{1}, \bar{u}+\zeta_{t_{2}} z_{2}, t\right)$. For the sake of simplicity we denote $F_{t ; \bar{u}}:=F_{t ; \bar{u} ; t, t}$. Thus

$$
F_{t ; \bar{u}}(z)=f\left(\xi_{t} z_{1}, \bar{u}+\zeta_{t} z_{2}, t\right),
$$

and the Frechet derivative of $F_{t ; \bar{u}}(z)$ is determined by

$$
F_{t ; \bar{u}}^{\prime}(z) w=\frac{\partial f}{\partial y}\left(\xi_{t} z_{1}, \bar{u}+\zeta_{t} z_{2}, t\right) \xi_{t} w_{1}+\frac{\partial f}{\partial x}\left(\xi_{t} z_{1}, \bar{u}+\zeta_{t} z_{2}, t\right) \zeta_{t} w_{2}
$$

where $w=\left(w_{1}^{T}, w_{2}^{T}\right)^{T} \in \mathbb{R}^{m}, w_{1} \in \mathbb{R}^{r}, w_{2} \in \mathbb{R}^{m-r}$.

Consider an equation

$$
F_{t ; \bar{u}}^{\prime}(z) w=q
$$


6 On discrete analogues of nonlinear implicit differential equations

where $q \in \mathbb{R}^{m}$, or equivalently,

$$
\frac{\partial f}{\partial y}\left(\xi_{t} z_{1}, \bar{u}+\zeta_{t} z_{2}, t\right) \xi_{t} w_{1}+\frac{\partial f}{\partial x}\left(\xi_{t} z_{1}, \bar{u}+\zeta_{t} z_{2}, t\right) \zeta_{t} w_{2}=q
$$

Observing that $\xi_{t} w_{1} \in \operatorname{Im} P(t), \zeta_{t} w_{2} \in \operatorname{Im} Q(t)$, hence $P(t) \xi_{t} w_{1}=\xi_{t} w_{1}, Q(t) \zeta_{t} w_{2}=\zeta_{t} w_{2}$, from (2.26) we find

$$
\frac{\partial f}{\partial y}\left(\xi_{t} z_{1}, \bar{u}+\zeta_{t} z_{2}, t\right) \xi_{t} w_{1}+\frac{\partial f}{\partial x}\left(\xi_{t} z_{1}, \bar{u}+\zeta_{t} z_{2}, t\right) Q(t) \zeta_{t} w_{2}=q .
$$

Taking into account the relation (2.10) and

$$
G^{-1}(y, x, t) \frac{\partial f}{\partial y}(y, x, t)=P(t)
$$

from (2.27) we get

$$
P(t) \xi_{t} w_{1}+Q(t) \zeta_{t} w_{2}=G^{-1}\left(\xi_{t} z_{1}, \bar{u}+\zeta_{t} z_{2}, t\right) q
$$

Multiplying both sides of (2.29) by $P(t)$ and $Q(t)$, respectively we find

$$
\begin{aligned}
& \xi_{t} w_{1}=P(t) G^{-1}\left(\xi_{t} z_{1}, \bar{u}+\zeta_{t} z_{2}, t\right) q, \\
& \zeta_{t} w_{2}=Q(t) G^{-1}\left(\xi_{t} z_{1}, \bar{u}+\zeta_{t} z_{2}, t\right) q .
\end{aligned}
$$

Thus (2.25) has a unique solution $w=\left(w_{1}^{T}, w_{2}^{T}\right)^{T}$. Moreover,

$$
\|w\| \leqslant c\left(\left\|w_{1}\right\|+\left\|w_{2}\right\|\right) \leqslant \widetilde{c}\|q\|
$$

for some positive constant $\tilde{c}$ since $G^{-1}\left(\xi_{t} z_{1}, \bar{u}+\zeta_{t} z_{2}, t\right), P(t), Q(t), \xi_{t}^{-1}, \zeta_{t}^{-1}$ are uniformly bounded. It follows that

$$
\left\|\left[F_{t ; \bar{u}}^{\prime}(z)\right]^{-1}\right\| \leqslant \tilde{c} .
$$

By the H'adamard theorem on homeomorphism (see $[4,10]), F_{t ; \bar{u}}$ is a homeomorphism between $\mathbb{R}^{m}$ and $\mathbb{R}^{m}$. For fixed $\bar{u} \in \mathbb{R}^{m}$ and $t \in J$, the equation

$$
F_{t ; \bar{u}}(z)=0
$$

has a unique solution

$$
z=\varphi(\bar{u}, t)=\left(\varphi_{1}^{T}(\bar{u}, t), \varphi_{2}^{T}(\bar{u}, t)\right)^{T}
$$

where $\varphi_{1}(\bar{u}, t) \in \mathbb{R}^{r}, \varphi_{2}(\bar{u}, t) \in \mathbb{R}^{m-r}$. Moreover, by the implicit function theorem, $\varphi(\bar{u}, t)$ is continuously differentiable in $\bar{u}$ and continuous in $t$ and

$$
\varphi_{\bar{u}}^{\prime}(\bar{u}, t)=-\left[F_{t ; \bar{u}}^{\prime}(z)\right]^{-1} \frac{\partial f}{\partial x}\left(\xi_{t} z_{1}, \bar{u}+\zeta_{t} z_{2}, t\right)
$$


The last relation shows that $\varphi_{\bar{u}}^{\prime}(\bar{u}, t)$ is uniformly bounded because $\left[F_{t ; \bar{u}}^{\prime}(z)\right]^{-1}$ is uniformly bounded by $(2.32)$ and $(\partial f / \partial x)(y, x, t)$ is uniformly bounded by assumption.

The application of the above mentioned arguments to (2.20) gives

$$
\begin{aligned}
P(t) x^{\prime}(t) & =\xi_{t} \varphi_{1}(u(t), t), \\
Q(t) x(t) & =\zeta_{t} \varphi_{2}(u(t), t) .
\end{aligned}
$$

On the other hand,

$$
\begin{aligned}
u^{\prime}(t) & =[P(t) x(t)]^{\prime}=P^{\prime}(t) x(t)+P(t) x^{\prime}(t)=P^{\prime}(t) x(t)+\xi_{t} \varphi_{1}(u(t), t) \\
& =P^{\prime}(t)[P(t) x(t)+Q(t) x(t)]+\xi_{t} \varphi_{1}(u(t), t) .
\end{aligned}
$$

Therefore, the IVP (2.1), (2.16) is equivalent to

$$
\begin{gathered}
u^{\prime}(t)=P^{\prime}(t) u(t)+P^{\prime}(t) \zeta_{t} \varphi_{2}(u(t), t)+\xi_{t} \varphi_{1}(u(t), t), \\
u\left(t_{0}\right)=P\left(t_{0}\right) x^{0}=: u^{0}, \\
x(t)=u(t)+\zeta_{t} \varphi_{2}(u(t), t) .
\end{gathered}
$$

Let

$$
\psi(u, t)=P^{\prime}(t) u+P^{\prime}(t) \zeta_{t} \varphi_{2}(u, t)+\xi_{t} \varphi_{1}(u, t)
$$

Clearly, $\psi$ is continuously differentiable in $u$ and continuous in $t$. Moreover, the partial derivative of $\psi$ w.r.t. $u$ is bounded, hence $\psi$ is Lipschitz continuous in $u$. It follows that the IVP (2.38), (2.39) and hence, the IVP (2.1), (2.16) has a unique solution on $J$.

Step 2. Now we return to the discretized IVP (2.6), (2.17). Arguing as in DAE case, we rewrite (2.6) as

$$
f\left(P_{n} \frac{x_{n+1}-x_{n}}{\tau}, u_{n}+Q_{n-1} x_{n}, t_{n}\right)=0, \quad n=\overline{0, N-1},
$$

where $u_{n}:=P_{n-1} x_{n}$. For a fixed $n \geq 0$ we consider the map

$$
F_{t_{n} ; u_{n} ; t_{n}, t_{n-1}}(z)=f\left(\xi_{t_{n}} z_{1}, u_{n}+\zeta_{t_{n-1}} z_{2}, t_{n}\right)
$$

where $t_{-1}:=t_{0}$.

Acting in the same manner as for (2.25), we realize that the equation

$$
F_{t_{n} ; u_{n} ; t_{n}, t_{n-1}}^{\prime}(z) w=q
$$

where $w=\left(w_{1}^{T}, w_{2}^{T}\right)^{T}$ and $w_{1} \in \mathbb{R}^{r}, w_{2} \in \mathbb{R}^{m-r}$, has the form

$$
\frac{\partial f}{\partial y}\left(\xi_{t_{n}} z_{1}, u_{n}+\zeta_{t_{n-1}} z_{2}, t_{n}\right) \xi_{t_{n}} w_{1}+\frac{\partial f}{\partial x}\left(\xi_{t_{n}} z_{1}, u_{n}+\zeta_{t_{n-1}} z_{2}, t_{n}\right) \zeta_{t_{n-1}} w_{2}=q .
$$


8 On discrete analogues of nonlinear implicit differential equations

Since $Q_{n-1} \zeta_{t_{n-1}} z_{2}=\zeta_{t_{n-1}} z_{2}$ and $Q_{n-1, n} Q_{n, n-1}=Q_{n-1}$, where $Q_{n, n-1}:=V_{n} \widetilde{Q} V_{n-1}^{-1}$, we can rewrite the last equation as

$$
\frac{\partial f}{\partial y}\left(\xi_{t_{n}} z_{1}, u_{n}+\zeta_{t_{n-1}} z_{2}, t_{n}\right) \xi_{t_{n}} w_{1}+\frac{\partial f}{\partial x}\left(\xi_{t_{n}} z_{1}, u_{n}+\zeta_{t_{n-1}} z_{2}, t_{n}\right) Q_{n-1, n} Q_{n, n-1} \zeta_{t_{n-1}} w_{2}=q .
$$

Using the relations

$$
\begin{gathered}
\widetilde{G}_{n}^{-1}\left(\xi_{t_{n}} z_{1}, u_{n}+\zeta_{t_{n-1}} z_{2}\right) \frac{\partial f}{\partial y}\left(\xi_{t_{n}} z_{1}, u_{n}+\zeta_{t_{n-1}} z_{2}, t_{n}\right)=P_{n} \\
\widetilde{G}_{n}^{-1}\left(\xi_{t_{n}} z_{1}, u_{n}+\zeta_{t_{n-1}} z_{2}\right) \frac{\partial f}{\partial x}\left(\xi_{t_{n}} z_{1}, u_{n}+\zeta_{t_{n-1}} z_{2}, t_{n}\right) Q_{n-1, n}=Q_{n}
\end{gathered}
$$

where $\widetilde{G}_{n}(y, x):=(\partial f / \partial y)\left(y, x, t_{n}\right)+(\partial f / \partial x)\left(y, x, t_{n}\right) Q_{n-1, n}$, we reduce $(2.44)$ to the form

$$
P_{n} \xi_{t_{n}} w_{1}+Q_{n, n-1} \zeta_{t_{n-1}} w_{2}=\widetilde{G}_{n}^{-1}\left(\xi_{t_{n}} z_{1}, u_{n}+\zeta_{t_{n-1}} z_{2}\right) q
$$

Multiplying both sides of the last equation by $P_{n}$ and $Q_{n}$, respectively, and taking into account relations

$$
\begin{gathered}
P_{n} Q_{n, n-1}=O ; \quad P_{n} \xi_{t_{n}} w_{1}=\xi_{t_{n}} w_{1} ; \\
Q_{n, n-1} \zeta_{t_{n-1}} w_{2}=V_{n} V_{n-1}^{-1} Q_{n-1} \zeta_{t_{n-1}} w_{2}=V_{n} V_{n-1}^{-1} \zeta_{t_{n-1}} w_{2},
\end{gathered}
$$

we get

$$
\begin{aligned}
\xi_{t_{n}} w_{1} & =P_{n} \widetilde{G}_{n}^{-1}\left(\xi_{t_{n}} z_{1}, u_{n}+\zeta_{t_{n-1}} z_{2}\right) q, \\
\zeta_{t_{n-1}} w_{2} & =Q_{n-1, n} \widetilde{G}_{n}^{-1}\left(\xi_{t_{n}} z_{1}, u_{n}+\zeta_{t_{n-1}} z_{2}\right) q .
\end{aligned}
$$

Therefore, (2.44) has a unique solution. On the other hand, since $G^{-1}(y, x, t)$ is uniformly bounded, we can prove that $\widetilde{G}_{n}^{-1}\left(\xi_{t_{n}} z_{1}, u_{n}+\zeta_{t_{n-1}} z_{2}\right)$ is also uniformly bounded and hence, $\|w\| \leqslant \widetilde{c}_{1}\|q\|$, where $\widetilde{c}_{1}$ is a positive constant, therefore $\left[F_{t_{n} ; u_{n} ; t_{n}, t_{n-1}}^{\prime}(z)\right]^{-1}$ is uniformly bounded. Using similar ideas as those employed to reduce (2.20) to the system (2.38) and (2.40), we can apply the H'adamard theorem to (2.42) to get

$$
\begin{aligned}
P_{n} \frac{x_{n+1}-x_{n}}{\tau} & =\xi_{t_{n}} \varphi_{1}\left(u_{n}, t_{n}\right), \\
Q_{n-1} x_{n} & =\zeta_{t_{n-1}} \varphi_{2}\left(u_{n}, t_{n}\right),
\end{aligned}
$$

or

$$
P_{n} x_{n+1}=P_{n} x_{n}+\tau \xi_{t_{n}} \varphi_{1}\left(u_{n}, t_{n}\right) ; \quad Q_{n-1} x_{n}=\zeta_{t_{n-1}} \varphi_{2}\left(u_{n}, t_{n}\right)
$$

Using the identity

$$
P_{n} x_{n}=\left(P_{n}-P_{n-1}\right) P_{n-1} x_{n}+\left(P_{n}-P_{n-1}\right) Q_{n-1} x_{n}+P_{n-1} x_{n}
$$


we rewrite the IVP (2.6), (2.17) as

$$
\begin{gathered}
u_{n+1}=\left(P_{n}-P_{n-1}\right) u_{n}+\left(P_{n}-P_{n-1}\right) \zeta_{t_{n-1}} \varphi_{2}\left(u_{n}, t_{n}\right)+u_{n}+\tau \xi_{t_{n}} \varphi_{1}\left(u_{n}, t_{n}\right), \\
u_{0}=u^{0}:=P_{0} x^{0}, \\
x_{n}=u_{n}+\zeta_{t_{n-1}} \varphi_{2}\left(u_{n}, t_{n}\right), \quad n=\overline{0, N} .
\end{gathered}
$$

Step 3. Together with (2.54), (2.55) we consider the explicit Euler scheme for the inherent ODE (2.38)

$$
\begin{gathered}
\frac{\bar{u}_{n+1}-\bar{u}_{n}}{\tau}=P_{n}^{\prime} \bar{u}_{n}+P_{n}^{\prime} \zeta_{t_{n}} \varphi_{2}\left(\bar{u}_{n}, t_{n}\right)+\xi_{t_{n}} \varphi_{1}\left(\bar{u}_{n}, t_{n}\right), \quad n=\overline{0, N-1}, \\
\bar{u}_{0}=u^{0}:=P_{0} x^{0}
\end{gathered}
$$

where $P_{n}^{\prime}:=P^{\prime}\left(t_{n}\right)$, or

$$
\begin{gathered}
\bar{u}_{n+1}=\tau P_{n}^{\prime} \bar{u}_{n}+\tau P_{n}^{\prime} \zeta_{t_{n}} \varphi_{2}\left(\bar{u}_{n}, t_{n}\right)+\tau \xi_{t_{n}} \varphi_{1}\left(\bar{u}_{n}, t_{n}\right)+\bar{u}_{n} \quad(n=\overline{0, N-1}), \\
\bar{u}_{0}=u^{0} .
\end{gathered}
$$

From (2.40), (2.56), it follows that

$$
\begin{aligned}
x\left(t_{n}\right)-x_{n}= & u\left(t_{n}\right)+\zeta_{t_{n}} \varphi_{2}\left(u\left(t_{n}\right), t_{n}\right)-u_{n}-\zeta_{t_{n-1}} \varphi_{2}\left(u_{n}, t_{n}\right) \\
= & \left(u\left(t_{n}\right)-\bar{u}_{n}\right)+\zeta_{t_{n}}\left[\varphi_{2}\left(u\left(t_{n}\right), t_{n}\right)-\varphi_{2}\left(\bar{u}_{n}, t_{n}\right)\right]+\left(\zeta_{t_{n}}-\zeta_{t_{n-1}}\right) \varphi_{2}\left(\bar{u}_{n}, t_{n}\right) \\
& +\left(\bar{u}_{n}-u_{n}\right)+\zeta_{t_{n-1}}\left[\varphi_{2}\left(\bar{u}_{n}, t_{n}\right)-\varphi_{2}\left(u_{n}, t_{n}\right)\right] \quad(n=\overline{0, N}) .
\end{aligned}
$$

Clearly, the explicit Euler method for the IVP (2.38), (2.39) is convergent, that is,

$$
\left\|\bar{u}_{n}-u\left(t_{n}\right)\right\|=O(\tau), \quad n=\overline{0, N}
$$

Further, the partial derivative of $\varphi_{2}$ w.r.t. $u$ is uniformly bounded and $\zeta_{t}$ is continuous on $J$, therefore we get

$$
\left\|\zeta_{t_{n}}\left[\varphi_{2}\left(u\left(t_{n}\right), t_{n}\right)-\varphi_{2}\left(\bar{u}_{n}, t_{n}\right)\right]\right\|=O(\tau) \quad(n=\overline{0, N}) .
$$

On the other hand, since $\bar{u}_{n}$ is bounded, $\varphi_{2}$ is continuous and $\zeta_{t}$ is uniformly continuous on $J$, we come to the conclusion that if

$$
\left\|u_{n}-\bar{u}_{n}\right\| \longrightarrow 0 \quad(\tau \longrightarrow 0)
$$

then

$$
\left\|x\left(t_{n}\right)-x_{n}\right\| \longrightarrow 0 \quad(\tau \longrightarrow 0) .
$$


10 On discrete analogues of nonlinear implicit differential equations

From (2.54), (2.58) we have

$$
\begin{aligned}
u_{n+1}-\bar{u}_{n+1}= & \left(u_{n}-\bar{u}_{n}\right)+\left(P_{n}-P_{n-1}\right)\left(u_{n}-\bar{u}_{n}\right) \\
& +\tau \xi_{t_{n}}\left[\varphi_{1}\left(u_{n}, t_{n}\right)-\varphi_{1}\left(\bar{u}_{n}, t_{n}\right)\right] \\
& +\left(P_{n}-P_{n-1}\right) \zeta_{t_{n-1}}\left[\varphi_{2}\left(u_{n}, t_{n}\right)-\varphi_{2}\left(\bar{u}_{n}, t_{n}\right)\right] \\
& +\left(P_{n}-P_{n-1}-\tau P_{n}^{\prime}\right) \bar{u}_{n}+\left(P_{n}-P_{n-1}-\tau P_{n}^{\prime}\right) \zeta_{t_{n-1}} \varphi_{2}\left(\bar{u}_{n}, t_{n}\right) \\
& +\tau P_{n}^{\prime}\left(\zeta_{t_{n-1}}-\zeta_{t_{n}}\right) \varphi_{2}\left(\bar{u}_{n}, t_{n}\right)
\end{aligned}
$$

this implies that

$$
\begin{aligned}
\left\|u_{n+1}-\bar{u}_{n+1}\right\| \leqslant & \left\|u_{n}-\bar{u}_{n}\right\|+\left\|P_{n}-P_{n-1}\right\|\left\|u_{n}-\bar{u}_{n}\right\|+\tau \widetilde{L}_{1}\left\|u_{n}-\bar{u}_{n}\right\| \\
& +\tilde{L}_{2}\left\|P_{n}-P_{n-1}\right\|\left\|u_{n}-\bar{u}_{n}\right\|+\left\|P_{n}-P_{n-1}-\tau P_{n}^{\prime}\right\|\left\|\bar{u}_{n}\right\| \\
& +\left\|P_{n}-P_{n-1}-\tau P_{n}^{\prime}\right\|\left\|\zeta_{t_{n-1}} \varphi_{2}\left(\bar{u}_{n}, t_{n}\right)\right\| \\
& +\tau\left\|P_{n}^{\prime}\right\|\left\|\left(\zeta_{t_{n-1}}-\zeta_{t_{n}}\right) \varphi_{2}\left(\bar{u}_{n}, t_{n}\right)\right\|,
\end{aligned}
$$

where $\widetilde{L}_{1}, \widetilde{L}_{2}$ are positive constants satisfying

$$
\begin{array}{r}
\left\|\xi_{t_{n}}\left[\varphi_{1}\left(u_{n}, t_{n}\right)-\varphi_{1}\left(\bar{u}_{n}, t_{n}\right)\right]\right\| \leqslant \widetilde{L}_{1}\left\|u_{n}-\bar{u}_{n}\right\|, \\
\left\|\zeta_{t_{n-1}}\left[\varphi_{2}\left(u_{n}, t_{n}\right)-\varphi_{2}\left(\bar{u}_{n}, t_{n}\right)\right]\right\| \leqslant \widetilde{L}_{2}\left\|u_{n}-\bar{u}_{n}\right\| .
\end{array}
$$

Putting $\alpha_{n}:=\left\|u_{n}-\bar{u}_{n}\right\|, a_{n}:=1+\left\|P_{n}-P_{n-1}\right\|+\tau \tilde{L}_{1}+\tilde{L}_{2}\left\|P_{n}-P_{n-1}\right\|$ and observing that

$$
\begin{aligned}
\gamma_{n}:= & \left\|P_{n}-P_{n-1}-\tau P_{n}^{\prime}\right\|\left\|\bar{u}_{n}\right\|+\left\|P_{n}-P_{n-1}-\tau P_{n}^{\prime}\right\|\left\|\zeta_{t_{n-1}} \varphi_{2}\left(\bar{u}_{n}, t_{n}\right)\right\| \\
& +\tau\left\|P_{n}^{\prime}\right\|\left\|\left(\zeta_{t_{n-1}}-\zeta_{t_{n}}\right) \varphi_{2}\left(\bar{u}_{n}, t_{n}\right)\right\| \\
= & o(\tau) \quad(n=\overline{0, N}), \quad \alpha_{0}=0,
\end{aligned}
$$

we get the estimate

$$
\alpha_{n+1} \leqslant \sum_{k=0}^{n-1}\left(\prod_{i=k+1}^{n} a_{i}\right) \gamma_{k}+\gamma_{n} \quad(n \geq 0) .
$$

Since $a_{i} \leqslant 1+\tau L$, where $L$ is a positive constant, we have

$$
\prod_{i=k+1}^{n} a_{i} \leqslant(1+\tau L)^{n-k} \leqslant(1+\tau L)^{n} \leqslant e^{n \tau L} \leqslant e^{L\left(T-t_{0}\right)} .
$$

Thus we come to the estimate

$$
\alpha_{n+1} \leqslant n e^{L\left(T-t_{0}\right)} \max _{k} \gamma_{k}+\gamma_{n}=\frac{o(\tau)}{\tau}, \quad \text { that is, }\left\|u_{n}-\bar{u}_{n}\right\| \longrightarrow 0 \quad(\tau \longrightarrow 0),
$$

as desired. Theorem 2.3 is proved.

Theorems 2.2 and 2.3 for linear DAEs were proved in [1]. 


\section{Nonlinear IDEs obtained via discretization of PDAEs}

Consider the PDAE

$$
\begin{gathered}
A(x, t) \frac{\partial u}{\partial t}=B(x, t) \frac{\partial^{2} u}{\partial x^{2}}+f(u, x, t), \quad 0<x<l, 0<t<T, \\
u(x, 0)=\varphi(x), \quad 0<x<l, \\
u(0, t)=\psi_{1}(t), \quad u(l, t)=\psi_{2}(t), \quad 0 \leqslant t \leqslant T,
\end{gathered}
$$

where $A, B \in C\left(J_{1} \times J_{2}, \mathbb{R}^{k \times k}\right)$ with $J_{1}:=[0, l]$ and $J_{2}:=[0, T], f \in C\left(\mathbb{R}^{k} \times J_{1} \times J_{2}, \mathbb{R}^{k}\right)$ and the matrix $A(x, t)$ is singular for every $(x, t) \in J_{1} \times J_{2}$.

In this section we impose the following conditions:

(H1) $\operatorname{Ker} A(x, t)=\mathcal{N}(t) \forall(x, t) \in J_{1} \times J_{2}$, and there exists a smooth projection $Q \in$ $C^{1}\left(J_{2}, \mathbb{R}^{k \times k}\right)$ onto $\operatorname{Ker} A(x, t)$, that is, $Q^{2}(t)=Q(t)$ and $\operatorname{Im} Q(t)=\mathcal{N}(t) \forall t \in J_{2}$. Moreover, $\operatorname{dim} \mathcal{N}(t) \equiv k-r$, where $1 \leqslant r \leqslant k-1$,

(H2) the matrices

$$
G(x, t):=A(x, t)+B(x, t) Q(t), \quad(x, t) \in J_{1} \times J_{2},
$$

are nonsingular,

(H3) there exists a positive constant $L$ such that

$$
\|f(\xi, x, t)-f(\zeta, x, t)\| \leqslant L\|\xi-\zeta\|
$$

for any $\xi, \zeta \in \mathbb{R}^{k}$ and $(x, t) \in J_{1} \times J_{2}$.

Now let $J_{1, h}=\left\{x_{0}<x_{1}<\cdots<x_{M}\right\}$ and $J_{2, \tau}=\left\{t_{0}<t_{1}<\cdots<t_{N}\right\}$ denote uniform partitions of $J_{1}$ and $J_{2}$, respectively, that is, $x_{m}:=m h(m=\overline{0, M})$ with $h=l / M$ and $t_{n}:=n \tau$, $n=\overline{0, N}$, where $\tau=T / N$. Put $A_{m n}:=A\left(x_{m}, t_{n}\right), B_{m n}:=B\left(x_{m}, t_{n}\right), G_{m n}:=G\left(x_{m}, t_{n}\right)(m=$ $\overline{0, M}, n=\overline{0, N}), \mathcal{N}_{n}:=\mathcal{N}\left(t_{n}\right)(n=\overline{0, N})$.

We are interested in the following discretized problem for (3.1):

$$
\begin{array}{r}
A_{m n} \frac{u_{m, n+1}-u_{m n}}{\tau}=B_{m n} \frac{u_{m+1, n}-2 u_{m n}+u_{m-1, n}}{h^{2}}+f\left(u_{m n}, x_{m}, t_{n}\right) \\
(m=\overline{1, M-1}, n=\overline{0, N-1),} \\
u_{m 0}=\varphi_{m} \quad(m=\overline{1, M-1}) ; \quad u_{0 n}=\psi_{1, n}, \quad u_{M n}=\psi_{2, n} \quad(n=\overline{0, N}),
\end{array}
$$

where $\varphi_{m}:=\varphi\left(x_{m}\right)(m=\overline{1, M-1}) ; \psi_{1, n}:=\psi_{1}\left(t_{n}\right), \psi_{2, n}:=\psi_{2}\left(t_{n}\right), n=\overline{0, N}$.

The reader interested in discretization methods for PDAEs should refer to $[7,8]$ and references therein. Let

$$
\mathscr{S}(x, t):=\left\{\xi \in \mathbb{R}^{k}: B(x, t) \xi \in \operatorname{Im} A(x, t)\right\} .
$$

Then the nonsingularity of $G(x, t)$ is equivalent to the condition

$$
\mathscr{S}(x, t) \cap \mathcal{N}(t)=\{0\} \quad \forall(x, t) \in J_{1} \times J_{2} .
$$


Since condition (3.7) is independent of the choice of $Q(t)$, the nonsingularity of $G(x, t)$ does not depend on the choice of $Q(t)$. For simplicity, we can choose orthogonal projections $Q(t)$ on $\mathcal{N}(t)$ and $Q(t)=V(t) \widetilde{Q} V^{T}(t)$, where $\widetilde{Q}=\operatorname{diag}\left(O_{r}, I_{k-r}\right)$ and $V(t)$ is an orthogonal matrix, that is,

$$
V(t) V^{T}(t)=V^{T}(t) V(t)=I \quad \forall t \in J_{2}
$$

Let $V_{n}:=V\left(t_{n}\right) ; Q_{n}:=Q\left(t_{n}\right), P_{n}:=I-Q_{n}(n=\overline{0, N})$ and $V_{-1}:=V_{0} ; Q_{-1}:=Q_{0} ; \mathcal{N}_{-1}:=$ $\mathcal{N}_{0} ; P_{-1}:=P_{0}$. In what follows, the norm of $\mathbb{R}^{k}$ is assumed Euclidean. From the smoothness of $Q(t)$ it can be proved that $V \in C^{1}\left(J_{2}, \mathbb{R}^{k \times k}\right)$. In this section, we also define the connecting operators as $Q_{n-1, n}:=V_{n-1} \widetilde{Q} V_{n}^{T}, n=\overline{0, N}$.

We introduce the matrix $\bar{G}_{m n}:=A_{m n}+B_{m n} Q_{n-1, n}$. Clearly,

$$
\bar{G}_{m n}=G_{m n}+B_{m n}\left(Q_{n-1, n}-Q_{n}\right)=G_{m n}\left[I+G_{m n}^{-1} B_{m n}\left(V_{n-1}-V_{n}\right) \tilde{Q} V_{n}^{T}\right]
$$

Further, we have

$$
\left\|G_{m n}^{-1} B_{m n}\left(V_{n-1}-V_{n}\right) \tilde{Q} V_{n}^{T}\right\| \leqslant c\left\|V_{n-1}-V_{n}\right\|
$$

where the generic constant $c$ is determined by the bounds of $G^{-1}, B, \widetilde{Q}$ and $V^{T}$. On the other hand,

$$
\left\|V_{n}-V_{n-1}\right\| \leqslant c_{1} \tau
$$

where $c_{1}=\max _{t \in J_{2}}\left\|V^{\prime}(t)\right\|$. Thus, we can conclude that for $\tau<\tau_{0}:=\left(c c_{1}\right)^{-1}$ the matrix $\bar{G}_{m n}$ has a uniformly bounded inverse, that is,

$$
\left\|\bar{G}_{m n}^{-1}\right\| \leqslant \bar{c}_{1} \quad \forall m=\overline{0, M}, \forall n=\overline{0, N} .
$$

Obviously,

$$
P_{n}=\bar{G}_{m n}^{-1} A_{m n} ; \quad Q_{n}=\bar{G}_{m n}^{-1} B_{m n} Q_{n-1, n} .
$$

Define

$$
\begin{array}{cl}
\alpha:=\frac{\tau}{h^{2}}, \quad v_{m n}:=P_{n-1} u_{m n} & (m=\overline{0, M}, n=\overline{0, N}), \\
\triangle_{h} u_{m n}:=u_{m+1, n}-2 u_{m n}+u_{m-1, n} & (m=\overline{1, M-1}, n=\overline{0, N}) .
\end{array}
$$

Clearly,

$$
P_{n-1} \triangle_{h} u_{m n}=\triangle_{h} v_{m n}=\triangle_{h} P_{n-1} u_{m n} \in \operatorname{Im} P_{n-1}
$$


Performing $P_{n} \bar{G}_{m n}^{-1}$ and $Q_{n} \bar{G}_{m n}^{-1}$ on both sides of (3.4), we get after a simple computation

$$
\begin{gathered}
v_{m, n+1}=P_{n} u_{m n}+\alpha P_{n} \bar{G}_{m n}^{-1} B_{m n} \triangle_{h} u_{m n}+\tau P_{n} \bar{G}_{m n}^{-1} f\left(u_{m n}, x_{m}, t_{n}\right), \\
u_{m 0}=\varphi_{m}, \\
\triangle_{h} u_{m n}=\left(I-Q_{n-1, n} \bar{G}_{m n}^{-1} B_{m n}\right) \triangle_{h} v_{m n}-h^{2} Q_{n-1, n} \bar{G}_{m n}^{-1} f\left(u_{m n}, x_{m}, t_{n}\right), \\
u_{0 n}=\psi_{1, n} ; \quad u_{M n}=\psi_{2, n} .
\end{gathered}
$$

Suppose for a fixed $n, u_{m n}$ is found ( $u_{m 0}=\varphi_{m}$ is given). Then the right-hand side of (3.16) is known. Thus we find $v_{m, n+1}(m=\overline{1, M-1})$. Besides, $v_{0, n+1}=P_{n} u_{0, n+1}=P_{n} \psi_{1, n+1}$, $v_{M, n+1}=P_{n} u_{M, n+1}=P_{n} \psi_{2, n+1}$.

For computing $u_{m, n+1}$ we consider the equation

$$
\triangle_{h} u_{m, n+1}=-h^{2} Q_{n, n+1} \bar{G}_{m, n+1}^{-1} f\left(u_{m, n+1}, x_{m}, t_{n+1}\right)+q_{m, n+1}, \quad m=\overline{1, M-1},
$$

where $q_{m, n+1}:=\left(I-Q_{n, n+1} \bar{G}_{m, n+1}^{-1} B_{m, n+1}\right) \triangle_{h} v_{m, n+1}$ is known. Introducing the notations

$$
\left.\begin{array}{c}
\bar{u}:=\left(u_{1, n+1}^{T}, \ldots, u_{M-1, n+1}^{T}\right)^{T}, \\
F:=\left(\begin{array}{ccccccc}
-2 I & I & O & \cdots & O & O & O \\
I & -2 I & I & \ldots & O & O & O \\
O & I & -2 I & \cdots & O & O & O \\
\vdots & \vdots & \vdots & \ddots & \vdots & \vdots & \vdots \\
O & O & O & \cdots & -2 I & I & O \\
O & O & O & \cdots & I & -2 I & I \\
O & O & O & \cdots & O & I & -2 I
\end{array}\right), \\
Q_{n, n+1} \bar{G}_{1, n+1}^{-1} f\left(u_{1, n+1}, x_{1}, t_{n+1}\right) \\
\vdots \\
Q_{n, n+1} \bar{G}_{M-1, n+1}^{-1} f\left(u_{M-1, n+1}, x_{M-1}, t_{n+1}\right)
\end{array}\right)+\left(\begin{array}{c}
q_{1, n+1} \\
\vdots \\
q_{M-1, n+1}
\end{array}\right) .
$$

Then (3.20) can be rewritten as

$$
E \bar{u}=F(\bar{u})
$$

We will prove that (3.22) has a unique solution, which can be approximated by iterations

$$
E \bar{u}^{(v+1)}=F\left(\bar{u}^{(v)}\right), \quad v=0, \ldots, \mu-1,
$$

where $\bar{u}^{(0)} \in \mathbb{R}^{k(M-1)}$ is an arbitrary vector and $\mu$ is a certain positive integer. 
14 On discrete analogues of nonlinear implicit differential equations

For this purpose we endow the space $\mathbb{R}^{k(M-1)}$ by the Euclidean norm, that is, if $\bar{z}=$ $\left(z_{1}^{T}, \ldots, z_{M-1}^{T}\right)^{T} \in \mathbb{R}^{k(M-1)}$ then $\|\bar{z}\|=\left(\sum_{m=1}^{M-1}\left\|z_{m}\right\|^{2}\right)^{1 / 2}$.

Using the Gaussian elimination we can reduce $E$ to the form

$$
U=\left(\begin{array}{ccccccc}
-2 I & I & O & \cdots & O & O & O \\
O & -\frac{3}{2} I & I & \cdots & O & O & O \\
O & O & -\frac{4}{3} I & \cdots & O & O & O \\
\vdots & \vdots & \vdots & \ddots & \vdots & \vdots & \vdots \\
O & O & O & \cdots & -\frac{M-2}{M-3} I & I & O \\
O & O & O & \cdots & O & -\frac{M-1}{M-2} I & I \\
O & O & O & \cdots & O & O & -\frac{M}{M-1} I
\end{array}\right) .
$$

Clearly, $\operatorname{det}(U)=(-1)^{k(M-1)} M^{k}$, hence $\operatorname{det}(E) \neq 0$, that is, $E$ is nonsingular.

Now we are able to state the existence and uniqueness theorem for problem (3.4)(3.5).

Theorem 3.1. Under the hypotheses (H1)-(H3) the discretized problem (3.4)-(3.5) has a unique solution, provided $h$ is sufficiently small and $\tau=\alpha h^{2}, \alpha=$ const.

Proof. From the above mentioned argument we see that the problem of finding solution of system (3.4)-(3.5) when $\tau, h$ are sufficiently small is reduced to the fixed-point problem

$$
\bar{u}=H(\bar{u}),
$$

where $H:=E^{-1} F$.

For any $\bar{u}, \bar{z} \in \mathbb{R}^{k(M-1)}$, we have

$$
\begin{aligned}
\|H(\bar{u})-H(\bar{z})\| & \leqslant h^{2}\left\|E^{-1}\right\|\left(\sum_{m=1}^{M-1}\left\|Q_{n, n+1} \bar{G}_{m, n+1}^{-1}\left(f\left(\bar{u}_{m}, x_{m}, t_{n+1}\right)-f\left(\bar{z}_{m}, x_{m}, t_{n+1}\right)\right)\right\|^{2}\right)^{1 / 2} \\
& \leqslant \bar{c}_{1} h^{2}\left\|E^{-1}\right\|\left(\sum_{m=1}^{M-1}\left\|f\left(\bar{u}_{m}, x_{m}, t_{n+1}\right)-f\left(\bar{z}_{m}, x_{m}, t_{n+1}\right)\right\|^{2}\right)^{1 / 2} \\
& \leqslant \bar{c}_{1} h^{2}\left\|E^{-1}\right\| L\left(\sum_{m=1}^{M-1}\left\|\bar{u}_{m}-\bar{z}_{m}\right\|^{2}\right)^{1 / 2} \\
& =\left(\bar{c}_{1} L\left\|E^{-1}\right\|\right) h^{2}\|\bar{u}-\bar{z}\| .
\end{aligned}
$$




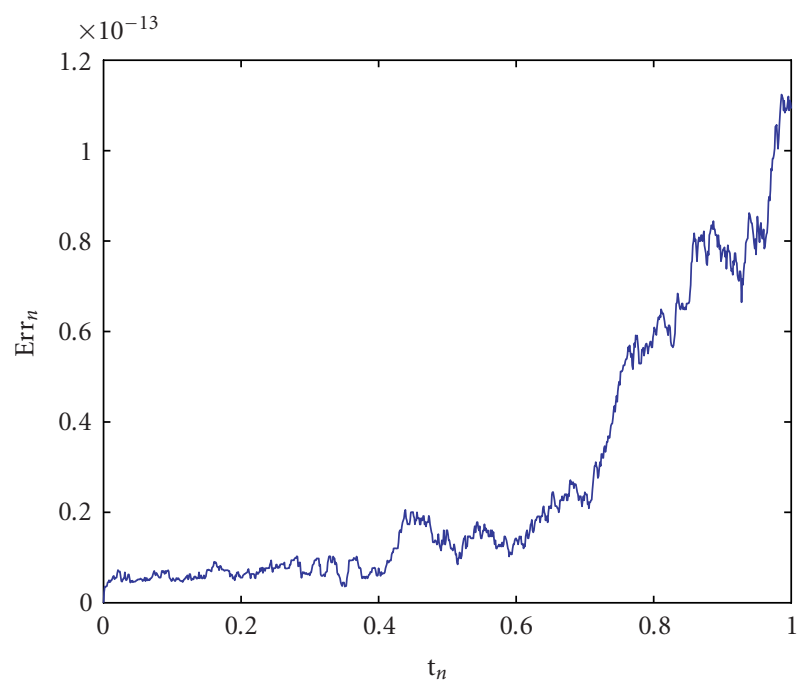

Figure 4.1. (Example 4.1) $h=0.05, \tau=0.001$.

Thus, for a sufficiently small $h>0, H$ is a contraction and the Banach theorem ensures the desired conclusions.

We end this section by recalling the sweeping method [3] applied to (3.23). Note that, (3.23) can be rewritten as

$$
\begin{array}{r}
\triangle_{h} u_{m, n+1}^{(\nu+1)}=-h^{2} Q_{n, n+1} \bar{G}_{m, n+1}^{-1} f\left(u_{m, n+1}^{(\nu)}, x_{m}, t_{n+1}\right)+q_{m, n+1}=: g_{m, n+1}^{(\nu)} \\
(m=\overline{1, M-1}, n=\overline{0, N-1}) .
\end{array}
$$

Firstly, we define $d_{m, n+1}^{(v)}(m=\overline{1, M-1})$ by the formulae

$$
d_{m, n+1}^{(\nu)}=\frac{m-1}{m} d_{m-1, n+1}^{(\nu)}+g_{m, n+1}^{(\nu)} \quad(m=\overline{2, M-1}) ; \quad d_{1, n+1}^{(v)}=g_{1, n+1}^{(\nu)}-\psi_{1, n+1} .
$$

Then $u_{m, n+1}^{(\nu+1)}(m=\overline{1, M-1})$ can be computed by the back substitution, namely

$$
u_{m, n+1}^{(\nu+1)}=\frac{m}{m+1}\left(u_{m+1, n+1}^{(\nu+1)}-d_{m, n+1}^{(\nu)}\right) \quad(m=\overline{M-1,1}) ; \quad u_{M, n+1}^{(\nu+1)}=\psi_{2, n+1} .
$$

\section{Numerical examples}

In this section, we consider two numerical examples. The computation was carried out in Matlab 7.0 on a Pentium IV 2.8 GHz running Windows NT.

The number of iterations and the initial approximation for the fixed-point iteration (3.23) in both examples are $\mu=50$ and $\bar{u}^{(0)}=0$, respectively. 


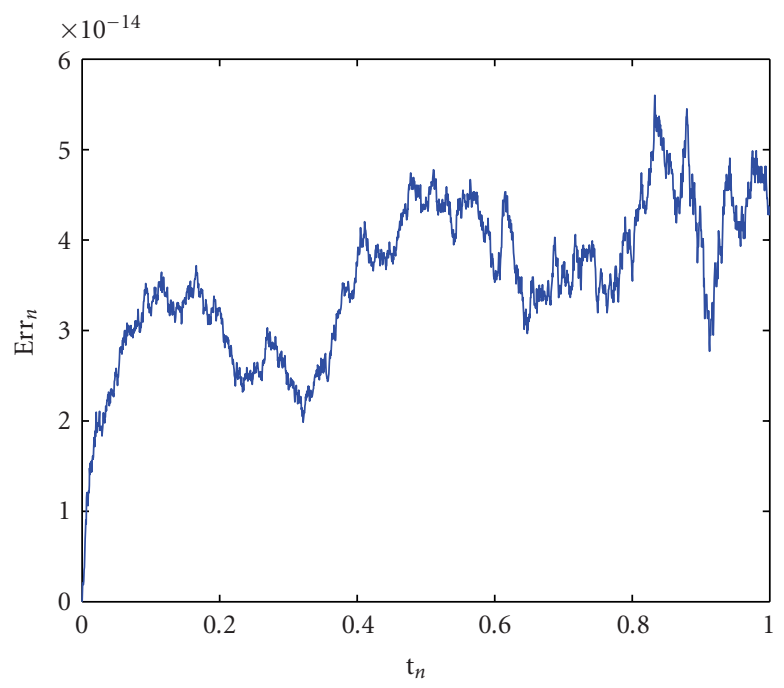

Figure 4.2. (Example 4.2) $h=0.05, \tau=0.0005$.

Figures 4.1 and 4.2 show the maximal values of the local error between the exact solution of (3.1) and the approximate solution determined by (3.23), that is,

$$
\operatorname{Err}_{n}:=\max _{0 \leqslant m \leqslant M}\left\|u_{m n}^{(\mu)}-u\left(x_{m}, t_{n}\right)\right\|, \quad n=\overline{0, N}
$$

for Examples 4.1 and 4.2, respectively.

Performing computation with different stepsizes $\tau$ and $h$ we observe that the convergence of the explicit scheme (3.4), (3.5) depends on the ratio $\tau / h^{2}$.

Example 4.1. Consider the PDAE (3.1) with the following data:

$$
\begin{gathered}
A(x, t)=\left(\begin{array}{cc}
x & -x(t+1) \\
1 & -(t+1)
\end{array}\right) ; \quad B(x, t)=\left(\begin{array}{cc}
0 & -(t+1) \\
x & 0
\end{array}\right) ; \\
f(u, x, t)=\left(\begin{array}{c}
\sin u_{1}+\sin x-x(t+1) \\
\cos u_{2}+\cos t-t-1
\end{array}\right) ; \\
\varphi(x)=\left(\begin{array}{c}
\pi+x \\
\pi
\end{array}\right) ; \quad \psi_{1}(t)=\left(\begin{array}{c}
\pi \\
\pi+t
\end{array}\right), \quad \psi_{2}(t)=\left(\begin{array}{c}
\pi+1 \\
\pi+t
\end{array}\right),
\end{gathered}
$$

where $u=\left(u_{1}, u_{2}\right)^{T} \in \mathbb{R}^{2}$, and $J_{1}=J_{2}=[0,1]=: J$.

Obviously, $u(x, t)=\left(\begin{array}{c}\pi+x \\ \pi+t\end{array}\right)$ is an exact solution of the PDAE (3.1) with data (4.2). Since $\operatorname{Ker} A(x, t)=\operatorname{span}\left\{(t+1,1)^{T}\right\}=: \mathcal{N}(t) \forall(x, t) \in[0,1]^{2}$ we can define $Q(t)=\left(\begin{array}{cc}0 & t+1 \\ 0 & 1\end{array}\right)$. Clearly, $Q \in C^{1}\left(J, \mathbb{R}^{2 \times 2}\right), Q^{2}(t)=Q(t)$ and $\operatorname{Im} Q(t)=\mathcal{N}(t) \forall t \in J$. Thus, we obtain

$$
G(x, t):=A(x, t)+B(x, t) Q(t)=\left(\begin{array}{cc}
x & -(x+1)(t+1) \\
1 & (x-1)(t+1)
\end{array}\right) .
$$


The nonsingularity of $G(x, t)$ follows from the fact that $\operatorname{det} G(x, t)=\left(x^{2}+1\right)(t+1)>0$ for all $(x, t) \in J \times J$. On the other hand, we have

$$
\begin{aligned}
\|f(\xi, x, t)-f(\zeta, x, t)\| & =\left\|\left(\sin \xi_{1}-\sin \zeta_{1}, \cos \xi_{2}-\cos \zeta_{2}\right)^{T}\right\| \\
& =\left(\left|\sin \xi_{1}-\sin \zeta_{1}\right|^{2}+\left|\cos \xi_{2}-\cos \zeta_{2}\right|^{2}\right)^{1 / 2} \\
& \leqslant\left(\left|\xi_{1}-\zeta_{1}\right|^{2}+\left|\xi_{2}-\zeta_{2}\right|^{2}\right)^{1 / 2} \\
& =\|\xi-\zeta\|
\end{aligned}
$$

where $\xi=\left(\xi_{1}, \xi_{2}\right)^{T}, \zeta=\left(\zeta_{1}, \zeta_{2}\right)^{T} \in \mathbb{R}^{2}$. Therefore, the conditions (H1)-(H3) are satisfied. Theorem 3.1 ensures the unique solvability of the discretized problem (3.4)-(3.5) with data (4.2), provided $h$ and $\tau$ are sufficiently small.

Example 4.2. In the second example we consider the PDAE (3.1) with the data:

$$
\begin{gathered}
A(x, t)=\left(\begin{array}{ccc}
t+1 & -x & x t \\
0 & 1 & -t \\
-x^{2} & 0 & 0
\end{array}\right) ; \quad B(x, t)=\left(\begin{array}{ccc}
x^{2}+1 & 0 & 0 \\
0 & 2 & 0 \\
0 & 0 & t+1
\end{array}\right) ; \\
f(u, x, t)=\left(\begin{array}{c}
\cos u_{1}+\sin x-x \\
\sin u_{2}+\sin t+1 \\
\left|u_{3}\right|-x
\end{array}\right) ; \\
\varphi(x)=\left(\begin{array}{c}
\pi / 2+x \\
\pi \\
x
\end{array}\right) ; \quad \psi_{1}(t)=\left(\begin{array}{c}
\pi / 2 \\
\pi+t \\
0
\end{array}\right), \quad \psi_{2}(t)=\left(\begin{array}{c}
\pi / 2+1 \\
\pi+t \\
1
\end{array}\right),
\end{gathered}
$$

where $u=\left(u_{1}, u_{2}, u_{3}\right)^{T} \in \mathbb{R}^{3}$, and $J_{1}=J_{2}=[0,1]=: J$.

In this case $u(x, t)=\left(\begin{array}{c}\pi / 2+x \\ \pi+t \\ x\end{array}\right)$ is an exact solution. Now we verify the hypotheses (H1)(H3). Firstly, we have $\operatorname{Ker} A(x, t)=\operatorname{span}\left\{(0, t, 1)^{T}\right\}=: \mathcal{N}(t) \forall(x, t) \in J \times J$. Therefore, choosing

$$
Q(t)=\frac{1}{t^{2}+1}\left(\begin{array}{ccc}
0 & 0 & 0 \\
0 & t^{2} & t \\
0 & t & 1
\end{array}\right)
$$

then we can verify that $Q \in C^{1}\left(J, \mathbb{R}^{3 \times 3}\right), Q^{2}(t)=Q(t)$ and $\operatorname{Im} Q(t)=\mathcal{N}(t) \forall t \in J$. Next, calculating $G(x, t):=A(x, t)+B(x, t) Q(t)$, we find

$$
G(x, t)=\left(\begin{array}{ccc}
t+1 & -x & x t \\
0 & \left(3 t^{2}+1\right) /\left(t^{2}+1\right) & \left(t-t^{3}\right) /\left(t^{2}+1\right) \\
-x^{2} & \left(t^{2}+t\right) /\left(t^{2}+1\right) & (t+1) /\left(t^{2}+1\right)
\end{array}\right)
$$


and $\operatorname{det} G(x, t)=(t+1)^{2}+2 x^{3} t>0 \forall(x, t) \in[0,1]^{2}$. It follows that $G(x, t)$ is nonsingular for all $(x, t) \in J \times J$. Further,

$$
\begin{aligned}
\|f(\xi, x, t)-f(\zeta, x, t)\| & =\left\|\left(\cos \xi_{1}-\cos \zeta_{1}, \sin \xi_{2}-\sin \zeta_{2},\left|\xi_{3}\right|-\left|\zeta_{3}\right|\right)^{T}\right\| \\
& =\left(\left|\cos \xi_{1}-\cos \zeta_{1}\right|^{2}+\left|\sin \xi_{2}-\sin \zeta_{2}\right|^{2}+\left|\left(\left|\xi_{3}\right|-\left|\zeta_{3}\right|\right)\right|^{2}\right)^{1 / 2} \\
& \leqslant\left(\left|\xi_{1}-\zeta_{1}\right|^{2}+\left|\xi_{2}-\zeta_{2}\right|^{2}+\left|\xi_{3}-\zeta_{3}\right|^{2}\right)^{1 / 2} \\
& =\|\xi-\zeta\|,
\end{aligned}
$$

where $\xi=\left(\xi_{1}, \xi_{2}, \xi_{3}\right)^{T}, \zeta=\left(\zeta_{1}, \zeta_{2}, \zeta_{3}\right)^{T} \in \mathbb{R}^{3}$. Thus, all the conditions (H1)-(H3) are satisfied. Theorem 3.1 ensures that the discretized problem (3.4)-(3.5) with data (4.5) has a unique solution when $h$ and $\tau$ are sufficiently small.

\section{Conclusion}

Explicit schemes applied to nonlinear DAEs and PDAEs lead to nonlinear IDEs. In this paper the unique solvability of discretized problems obtained via discretization of nonlinear implicit differential equations is studied. The compatibility between index notions for nonlinear index-1 DAEs and nonlinear index-1 IDEs as well as the convergence of the explicit Euler method for nonlinear index-1 DAEs were established.

The investigation on the stability and convergence of an explicit scheme for degenerated parabolic equations is now in progress. Besides, the Floquet theory for linear index-1 IDEs and its applications to the stability theory for nonlinear IDEs has been established.

\section{Acknowledgment}

The authors would like to express their gratitude to the referees, whose careful reading and numerous comments led to an improvement in style and presentation of the paper.

\section{References}

[1] P. K. Anh, N. H. Du, and L. C. Loi, Connections between implicit difference equations and differential-algebraic equations, Acta Mathematica Vietnamica 29 (2004), no. 1, 23-39.

[2] P. K. Anh and H. T. N. Yen, On the solvability of initial-value problems for nonlinear implicit difference equations, Advances in Difference Equations 2004 (2004), no. 3, 195-200.

[3] N. S. Bakhvalov, N. P. Zhidkov, and G. M. Kobel'kov, Numerical Methods, Nauka, Moscow, 1987.

[4] M. S. Berger, Nonlinearity and Functional Analysis. Lectures on Nonlinear Problems in Mathematical Analysis, Pure and Applied Mathematics, Academic Press, New York; Harcourt Brace Jovanovich, London, 1977.

[5] E. Griepentrog and R. März, Differential-Algebraic Equations and Their Numerical Treatment, Teubner Texts in Mathematics, vol. 88, BSB B. G. Teubner, Leipzig, 1986.

[6] L. C. Loi, N. H. Du, and P. K. Anh, On linear implicit non-autonomous systems of difference equations, Journal of Difference Equations and Applications 8 (2002), no. 12, 1085-1105. 
[7] W. Lucht and K. Debrabant, On quasi-linear PDAEs with convection: applications, indices, numerical solution, Applied Numerical Mathematics 42 (2002), no. 1-3, 297-314.

[8] W. Lucht, K. Strehmel, and C. Eichler-Liebenow, Indexes and special discretization methods for linear partial differential algebraic equations, BIT 39 (1999), no. 3, 484-512.

[9] R. März, On linear differential-algebraic equations and linearizations, Applied Numerical Mathematics 18 (1995), no. 1-3, 267-292.

[10] J. M. Ortega and W. C. Rheinboldt, Iterative Solution of Nonlinear Equations in Several Variables, Academic Press, New York, 1970.

Pham Ky Anh: Department of Mathematics, Vietnam National University, Hanoi, Vietnam

E-mail address: anhpk@vnu.edu.vn

Le Cong Loi: Department of Mathematics, Vietnam National University, Hanoi, Vietnam E-mail address: loilc@vnu.edu.vn 